ROME prep. 1155/96

\title{
THEORETICAL REVIEW OF $B$-PHYSICS
}

\author{
G. Martinelli \\ Dip. di Fisica, Università degli Studi di Roma "La Sapienza" and \\ INFN, Sezione di Roma, P.le A.Moro 2, 00185, Rome, Italy
}

\begin{abstract}
Weak decays and mixing of $B$-hadrons play a special role in our understanding of the physics of the Standard Model and beyond. The measured amplitudes, however, result from a complicated interplay of weak and strong interaction effects. Understanding strong interaction dynamics, which becomes simpler for heavy quarks, is thus a fundamental part of the game. In this review, several theoretical aspects of $B$ dynamics which are relevant for current and future experimental measurements are discussed.
\end{abstract}




\section{Introduction}

Hadronic weak decays are a unique source of information on the physics of the Standard Model and beyond. Experimental measurements and theoretical studies of these decays may provide us the key for understanding still unsolved problems such as the reason for the existence of several fermion families, the origin of fermion masses and weak couplings and the mechanisms which give rise to $C P$ violation. Through quantum-loop effects, they are also a window which allows us to explore and constraint new physics effects at high energy scales still unaccessible to particle accellerators, such as those due to heavy supersymmetric particles. Unfortunately the physical amplitudes result from a complicated interplay of weak and strong interaction effects, which must be disentangled in order to extract the values of the fundamental parameters.

In this framework, $B$-hadron decays play a special role, since the $b$-quark belongs to the heaviest, and less "coupled", of the three known families and its partner, the top quark, has a mass which is of the order of the weak scale, $m_{t} \sim G_{F}^{-1 / 2}$. This observation, for example, has led to several speculations: that spontaneous symmetry breaking occurs via the Nambu-Jona Lasinio mechanism [1], that quark masses are dynamically determined by radiative corrections [2] or that the fundamental quark mass matrix, at large energy scales, is constrained to have very specific forms [3]. Another aspect which is specific to $B$-hadron decays is that the mass of the $b$-quark is much larger than the scale of strong interactions, $m_{b} \gg \Lambda_{Q C D}$. In this situation, QCD dynamics becomes much simpler and in many cases, by using the Heavy Quark Effective Theory (HQET) [4], we can obtain accurate theoretical predictions to compare with the experimental measurements. An example is given by the determination of the Cabibbo-Kobayashi-Maskawa (CKM) matrix element $\left|V_{c b}\right|$ from exclusive $\left(B \rightarrow D^{*} l \nu_{l}\right.$ and $\left.B \rightarrow D l \nu_{l}\right)$ and inclusive $\left(b \rightarrow X_{c} l \nu_{l}\right)$ semi-leptonic decays. Since, however, the mass of the $b$-quark is large, but not infinite, a related problem, to be discussed in the following, is that of controlling power corrections, i.e. corrections suppressed as inverse powers of the heavy quark mass.

In this article, I review several theoretical issues in the physics of $B$ decays which are relevant for current or future experimental measurements. A special attention is paid to the discussion of the systematic errors present in the different theoretical approaches, and of the possible progresses that we may expect in the near future. In preparing this talk, it has been unavoidable to make a selection of subjects. Among those for which new theoretical studies or new experimental measurements appeared this year, I have chosen those topics which look to me particularly interesting, and/or for which I have enough compentence to express a personal point of view. For lack of space, I am unable to cover here radiative decays and supersymmetric effects in FCNC processes. A discussion on these points can be found in the talks by A. Ali and A. Masiero at this workshop. For further information on other subjects related to $B$-physics, not covered here, the reader can also refer to some recent review papers 
by A. Ali [5], A. Buras [6] and M. Neubert [7].

This article is organized as follows. In sec. 2, a brief overview of leptonic decays, $B^{0}-\bar{B}^{0}$ mixing and $\mathrm{CP}$ violation is given; in sec. 3, $B$-meson exclusive semi-leptonic decays, and the extraction of $\left|V_{c b}\right|$ and $\left|V_{u b}\right|$, are discussed; some aspects of exclusive non-leptonic decays are reviewed in sec. 4 ; power corrections in the HQET are discussed in sec. 5; some considerations on inclusive semi-leptonic decays can be found in sec. 6; the present status, and unsolved problems, for $B$-hadron lifetimes will be summarized in sec. [7, followed by a brief conclusion, which contains also an outlook on possible improvements in the accuracy of the theoretical predictions, sec. 8 .

\section{$2 f_{B}, B^{0}-\bar{B}^{0}$ mixing and CP violation}

The leptonic decay width of the $B$ meson is given by

$$
\Gamma\left(B^{+} \rightarrow \tau^{+} \nu_{\tau}\right)=\frac{G_{F}^{2}\left|V_{u b}\right|^{2}}{8 \pi} M_{B}\left(1-\frac{M_{\tau}^{2}}{M_{B}^{2}}\right)^{2} M_{\tau}^{2} f_{B}^{2} .
$$

A precise knowledge of the leptonic decay constant $f_{B}$, analogous to $f_{\pi}$ in $\pi \rightarrow \mu \nu_{\mu}$ decays ⿹, would allow an accurate extraction of the CKM matrix element $\left|V_{u b}\right|$, which is actually known with a relative error of about $25 \%$, see below. One of the most precise determinations of $f_{B}$ is presently given by lattice QCD calculations and it is shown in table 1, together with other results for several physical quantities of interest for $B$-physics [8]- 10]. The error on $f_{B}$ reported in the table comes mainly from the extrapolation of the lattice results to the continuum limit and does not includes the systematic error due to the quenched approximation, which has been estimated to be of the order of 10-30\% [11]. A global theoretical error of $25 \mathrm{MeV}$, corresponding to a relative error of about $14 \%$, would provide us with an accurate determination of $\left|V_{u b}\right|$, through a measurement totally independent from the semi-leptonic channel, and with experimental and theoretical systematic effects different from that case. Even though the rate (which goes as the squared lepton mass) is much smaller, the $B^{+} \rightarrow \mu^{+} \nu_{\mu}$ leptonic decay channel is also quite interesting, because the much smaller number of events could be compensated by a much higher efficiency in detecting the final state charged lepton.

$f_{B}$ also enters in phenomenological analyses of the $B^{0}-\bar{B}^{0}$ mixing amplitudes, together with the so called renormalization group invariant $B$-parameter $\hat{B}_{B}$. The square of the mixing parameter $\xi=f_{B} \sqrt{\hat{B}_{B}}$ is in fact related to the matrix element of the renormalized $\Delta B=2$ Hamiltonian [12]. All theoretical calculations of $\hat{B}_{B}$ tend to give values very close to one for both the $B_{d}^{0}$ and the $B_{s}^{0}$ mesons, see table 11 and ref. [14 for QCD sum rules. Thus

\footnotetext{
${ }^{1}$ I use the normalization convention in which $f_{\pi}=132 \mathrm{MeV}$.
} 


\begin{tabular}{||c|c|c|c||}
\hline$f_{D}(\mathrm{MeV})$ & $f_{D_{s}}(\mathrm{MeV})$ & $f_{B}(\mathrm{MeV})$ & $f_{B_{s}} / f_{B}$ \\
\hline $205 \pm 15$ & $235 \pm 15$ & $175 \pm 25$ & $1.15 \pm 0.05$ \\
\hline$\hat{B}_{B_{d}}$ extrapolated & $\hat{B}_{B_{d}} \mathrm{HQET}$ & $\hat{B}_{B_{d}} / \hat{B}_{B_{s}}$ & $\xi_{d}(\mathrm{MeV})$ \\
\hline $1.40 \pm 0.10$ & $1.08 \pm 0.06 \pm 0.08$ & $1.01 \pm 0.01$ & $207 \pm 30$ \\
\hline
\end{tabular}

Table 1: Lattice predictions for several quantities of interest in heavy flavour physics. A detailed explanation of the methods used to compute these quantities, and of the evaluation of the systematic errors can be found in [8]-[10]. The numbers given here are my personal compilation of lattice results obtained from the numbers reported in refs. [8]-[10] and the results which can be found in ref. 113.

the strength of the mixing is essentially regulated by the meson decay constant. Taking into account the theoretical uncertainties, and the experimental errors on the relevant CKM matrix elements and on the $B$ lifetime, one predicts $\Delta M_{B_{d}}=0.55 \pm 0.13$ ps [15], to be compared with the experimental world average $\Delta M_{B_{d}}=0.464 \pm 0.018$ ps.

The value of $f_{B}$ is also important for $\mathrm{CP}$ violation in $B$ decays. It was realized long time ago [16] that a value of $\xi \sim 200 \mathrm{MeV}$, combined with a large value of the top mass, (in the following I will use $\left.m_{t}^{\overline{M S}}\left(m_{t}^{\overline{M S}}\right)=167 \pm 8 \mathrm{GeV}\right)$ leads to a large value of $\sin 2 \beta$, the parameter which controls $\mathrm{CP}$ violation in $B \rightarrow J / \psi K_{s}$ decays

$$
\mathcal{A}=\frac{N\left(B_{d}^{0} \rightarrow J / \psi K_{s}\right)(t)-N\left(\bar{B}_{d}^{0} \rightarrow J / \psi K_{s}\right)(t)}{N\left(B_{d}^{0} \rightarrow J / \psi K_{s}\right)(t)+N\left(\bar{B}_{d}^{0} \rightarrow J / \psi K_{s}\right)(t)}=\sin 2 \beta \sin \Delta M_{B_{d}} t
$$

This is shown in fig. 11 where the distribution of acceptable values of $\sin 2 \beta$, obtained from a combined analysis of the kaon CP violation parameter $\epsilon$ and of the $B_{d}^{0}-\bar{B}_{d}^{0}$ mixing rate $\Delta M_{B_{d}}$ is shown [15]. The histogram refers to the distribution of values of $\sin 2 \beta$ obtained by varying within their errors the values of the different experimental and theoretical quantities which enter in the theoretical predictions. The dashed histogram is obtained as the previous one, by imposing the further condition that $\xi=207 \pm 30 \mathrm{MeV}$. From the dashed distribution shown in the figure, one gets $\sin 2 \beta=0.68 \pm 0.10$, a value quite reassuring for future experiments at the $B$-factories.

The reliability of the lattice estimates of the meson decay constants is demonstrated by the fact that $f_{D_{s}}$, which was predicted long before the first experimental measurement [17], agrees with the present experimental world average from $D_{s} \rightarrow \mu \nu_{\mu}$ decays, $f_{D_{s}}=241 \pm 21 \pm 30$ $\mathrm{MeV}\left(f_{D_{s}} \sim 260 \mathrm{MeV}\right.$ if one includes the L3 measurement from $\left.D_{s} \rightarrow \tau \nu_{\tau}\right)$ [18]. Further, indirect information can be extracted from the experimental lower bound on the $B_{s}^{0}-\bar{B}_{s}^{0}$ mixing amplitude. Taking $\Delta M_{B_{s}} \geq 9.2 \mathrm{ps}$ from experiments and $f_{B_{s}} / f_{B}=1.15 \pm 0.05^{+0.15}$ from theory, where the last error is an estimate of the error coming from the quenched approximation \&, one obtains $f_{B} \geq 160 \mathrm{MeV}$ in good agreement with the direct theoretical

\footnotetext{
${ }^{2}$ In the ratio of the decay constants many systematic errors cancel.
} 


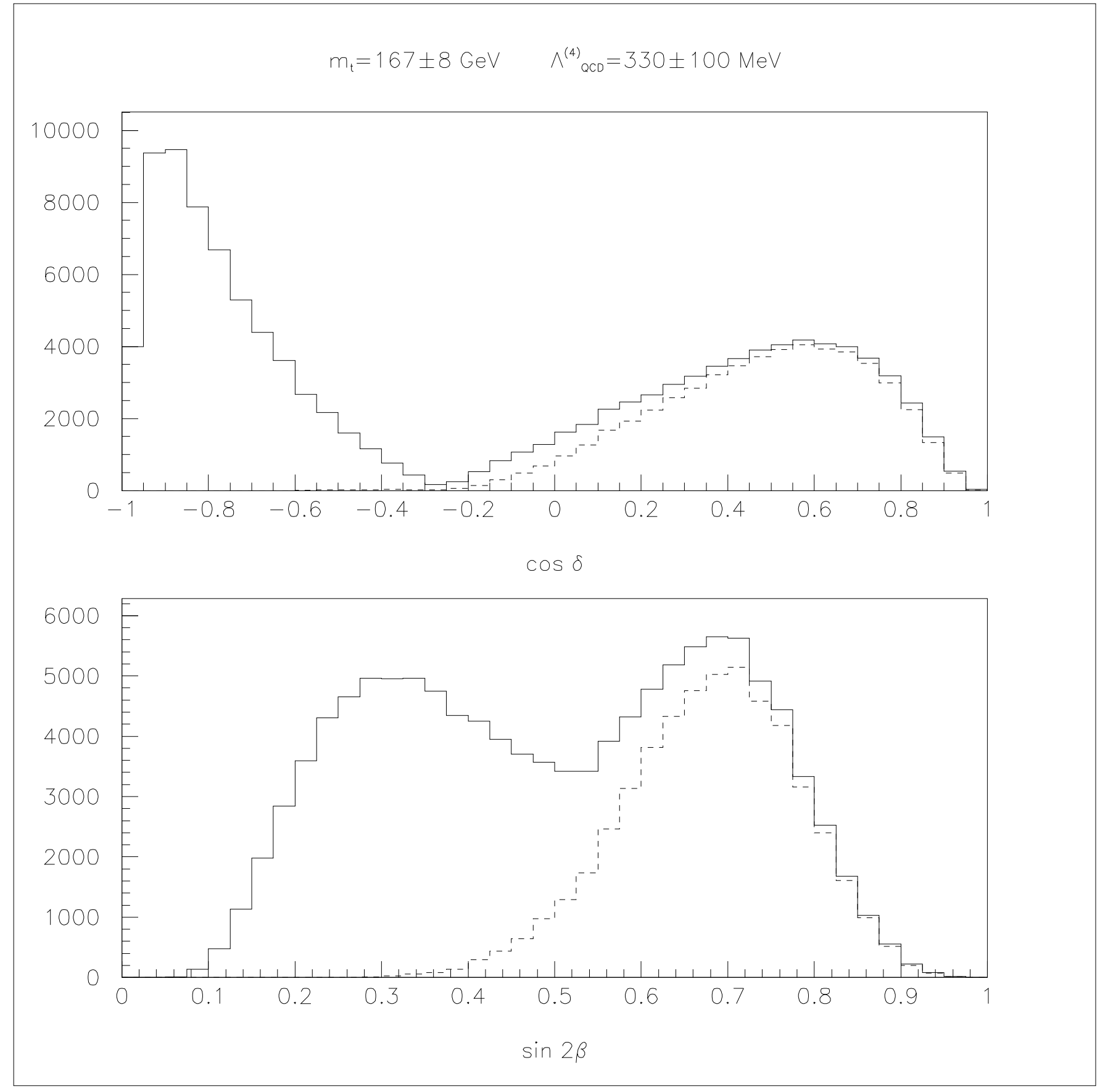

Figure 1: Distributions of values for $\cos \delta$ and $\sin 2 \beta$, for $m_{t}=(168 \pm 8)$ GeV. The values of the parameters are those used in the second of refs. 10]. The solid histograms are obtained without using the information coming from the experimental determination of $\Delta M_{B_{d}}$. The dashed ones uses $\Delta M_{B_{d}}=0.464 \pm 0.018$, with the condition $177 \mathrm{MeV} \leq \xi_{d}=f_{B_{d}} \sqrt{\hat{B}_{B_{d}}} \leq 237$ $M e V$. 
prediction [15].

I want to conclude this section with a comment on the "heavyness" of the charm quark. The HQET is not a model. It is QCD systematically expanded in powers of a small parameter $\mu_{Q C D} / m_{Q}$, where $m_{Q}$ is the quark mass and $\mu_{Q C D}$ stands for a scale of the order of the strong interaction scale. Thus if $\mu_{Q C D}=\Lambda_{Q C D} \sim 300 \mathrm{MeV}$, since $m_{\text {charm }} \sim 1.2-1.5 \mathrm{GeV}$, the expansion parameter is a small number even in the charm case. If, instead, $\mu_{Q C D}=$ $2 \pi f_{D} \sim 1.2 \mathrm{GeV}$ or of the order of the nucleon mass, the expansion parameter is of order one and the heavy quark expansion is expected to fail. Lattice studies of the behaviour of the meson decay constant $f_{M}$ as a function of the inverse meson mass [8]- [10] have shown that the expansion parameter is, at least in this case, of the order of $1 \mathrm{GeV}$. These results have been confirmed by calculations using QCD sum rules [14. Such a large value of $\mu_{Q C D}$ implies that power corrections are $\sim 80 \%-100 \%$ for the charmed meson decay constant, and $\sim 25 \%-30 \%$ in the $B$-meson case. Corrections of the same order are also expected, and were indeed found in lattice calculations, for the semi-leptonic form factors relevant in $B \rightarrow \pi$ and $B \rightarrow \rho$ decays [9]. A special, and probably unique, case where the application of the HQET to the charm is succesful, because of Luke's theorem [19], is represented by the semi-leptonic $B \rightarrow D$ and $D^{*}$ decays which are discussed in sec. 3. In all the other cases, as in the discussion of the charmed hadron lifetimes of sec. 7, there is strong experimental evidence that the charm is not heavy enough to make HQET applicable.

\section{Exclusive semi-leptonic decays}

In this section I will discuss some theoretical issues related to exclusive heavy-to-heavy and heavy-to-light semi-leptonic decays.

\section{1 $\quad V_{c b}$ from $B \rightarrow D^{*} l \nu_{l}$}

In heavy-to-heavy decays, a golden plated determination of $\left|V_{c b}\right|$ is given by the comparison of the differential decay rate for the process $B \rightarrow D^{*} l \nu_{l}$ to its theoretical expression f $^{\circ}$

$$
\begin{aligned}
\frac{d \Gamma}{d w} & =\frac{G_{F}^{2}}{48 \pi^{3}}\left(M_{B}-M_{D^{*}}\right)^{2} M_{D^{*}}^{3} \sqrt{w^{2}-1}(w+1)^{2} \\
& \times\left(1+\frac{4 w}{w+1} \frac{M_{B}^{2}-2 w M_{B} M_{D^{*}}+M_{D^{*}}^{2}}{\left(M_{B}-M_{D^{*}}\right)^{2}}\right)\left|V_{c b}\right|^{2} \mathcal{F}^{2}(w),
\end{aligned}
$$

\footnotetext{
${ }^{3}$ For lack of space I will not discuss the process $B \rightarrow D l \nu_{l}$ for which both measurements, and theoretical predictions, exist.
} 
where the variable $w$, related to the momentum trasfer $q$, is given by

$$
w=v_{B} \cdot v_{D^{*}}=\frac{E_{D^{*}}}{M_{D^{*}}}=\frac{M_{B}^{2}+M_{D^{*}}^{2}-q^{2}}{2 M_{B} M_{D^{*}}} .
$$

In the infinite mass limit, up to logarithmic corrections, $\mathcal{F}(w)$ tends to the Isgur-Wise function $\xi(w)$. When both $m_{b}$ and $m_{\text {charm }}$ become very large, the normalization of $\xi(w)$ at zero recoil is fixed by the conservation of the weak current, i.e. $\xi(w=1)=1$. In these limits then, up to the trivial kinematical factors appearing in eq. (4), a model independent extraction of $\left|V_{c b}\right|$ is possible. Two elements are important for an accurate determination of $\left|V_{c b}\right|$, however: the control of the corrections due to different effects at zero recoil, and the extrapolation of the rate to $w=1$.

i) The normalization of $\mathcal{F}(w=1)$ is modified by short-distance strong interactions effects, which can in principle be computed in perturbation theory, and by non-perturbative $1 / m_{Q}$ corrections (starting at second order by virtue of Luke's theorem [19]). We have then

$$
\mathcal{F}(w=1)=\eta_{A}\left(1+c_{2} \frac{\mu_{Q C D}^{2}}{m_{Q}^{2}}+\ldots\right)=\eta_{A}\left(1+\delta_{1 / m^{2}}\right) .
$$

A recent two-loop calculation has reduced the uncertainty coming from the perturbative corrections to less than $1 \%$, giving $\eta_{A}=0.960 \pm 0.007$ 20. The evaluation of $\delta_{1 / m^{2}}$ is more problematic, since this is a non-perturbative quantity. Two different approaches were followed in the past. On the one hand, a classification of all the $1 / m_{Q}^{2}$ local operators of the HQET contributing to $\delta_{1 / m^{2}}$ was made, and their matrix elements estimated in a model dependent way [21]. These estimates gave $-\delta_{1 / m^{2}}=(3 \pm 2) \%$. On the other hand, zerorecoil sum rules, which are in principle model-independent, gave a much larger correction corresponding to $-\delta_{1 / m^{2}}=5.5 \%-8 \%$ [22, 23]. After some controversy, M. Neubert, by using a "hybrid" method, found a "Solomonic" result, which is "not-in-disagreement" with both the values given above, namely $-\delta_{1 / m^{2}}=(5.5 \pm 2.5) \%$ [24. This prediction is currently adopted in all the estimates of the theoretical error on $\left|V_{c b}\right|$ extracted from the experimental measurements of the exclusive semi-leptonic decays.

A recent study of the accuracy of power corrections in effective theories, illustrated by several explicit examples, showed that the predictions from the zero recoil sum rules, though model independent, are potentially subject to large corrections coming from higher order perturbative terms which have not been computed yet 25]. The same arguments apply to the calculations of refs. [21, 24]. For this reason, and given the high accuracy reached by the experimental measurements, I believe that a further effort is needed to try to reduce, and to evaluate more realistically, the error on $\delta_{1 / m^{2}}$. This is very difficult because the deviations due to $\delta_{1 / m^{2}}$ are rather small, and no theoretical method, including lattice simulations, can control the uncertanties to a sufficient degree of accuracy. 
ii) Since the rate vanishes at $w=1$ (see eq. (3)) in order to extract $\mathcal{F}(w=1)$ an extrapolation of the data is necessary. This extrapolation is usually done by expanding the form factor around $w=1$

$$
\mathcal{F}(w)=\mathcal{F}(1)\left(1-\hat{\rho}^{2}(w-1)+\hat{c}(w-1)^{2}+\ldots\right) .
$$

The slope $\hat{\rho}$ and the curvature $\hat{c}$ can be fitted directly from the experimental measurements of the differential rate [26, 27]. It is, however, helpful for reducing the uncertainty of the extrapolation to have theoretical estimates of these parameters, for which several bounds, and calculations, obtained using different techniques, exist. The spread of the theoretical predictions for $\hat{\rho}$ is, unfortunately, still rather large [8], while for $\hat{c}$ there are only analyticity bounds which relate its value to that of $\hat{\rho}$ [28. In analogy to what is done experimentally, $\hat{\rho}$ has been extracted from lattice calculations by fitting the form factor computed in numerical simulations to eq. (6). This fitting procedure, however, introduces a rather large uncertainty in the final result [8]. I want to mention that a method to obtain directly the $\hat{\rho}$ and $\hat{c}$, without any fitting procedure, or perturbative calculation, has been proposed in ref. [29, but only a preliminary feasibility study of this technique has been done to date [30. Contrary to the case of $\delta_{1 / m^{2}}$, it is likely that more accurate theoretical results for $\hat{\rho}$ will be obtained in the near future.

As shown in fig. 2, the values of $\mathcal{F}(w=1)\left|V_{c b}\right|$ extracted by the different experiments are quite consistent. This is true in spite of the fact that the fitted values of $\hat{\rho}$ are somehow different [27]. From the world average of $\mathcal{F}(w=1)\left|V_{c b}\right|$, by assuming from the theory $\mathcal{F}(w=1)=0.91 \pm 0.03$ one obtains,

$$
\left|V_{c b}\right|=0.0382 \pm 0.0018 \pm 0.0012
$$

where the last error comes from the estimated theoretical uncertainty.

\section{2 $\quad V_{u b}$ from $B \rightarrow \rho l \nu_{l}$ and $B \rightarrow \pi l \nu_{l}$}

For heavy-to-light semi-leptonic decays, HQET is less useful since the normalization of the current is not fixed by symmetry. Still scaling laws can be established for $q^{2} \sim q_{\max }^{2}$. For example, up to logarithmic corrections, one finds [31, 32]

$$
f_{P}^{+}\left(\vec{p}_{\pi}\right)=A M_{P}^{1 / 2}\left(1+\frac{\delta}{M_{P}}+\ldots\right)
$$

where $f_{B}^{+}\left(\vec{p}_{\pi}\right)$ is the form factor relevant in $B \rightarrow \pi$ decays. $P$ denotes the heavy pseudoscalar meson, $M_{P}$ its mass, $\vec{p}_{\pi}$ the pion momentum and $A$ and $\delta$ are non perturbative quantities.

\footnotetext{
${ }^{4}$ Following ref. [7], I use here $\hat{\rho}$ to denote the physical slope, in order to distinguish it from $\rho$ which refers to the slope of the Isgur-Wise function $\xi$.
} 


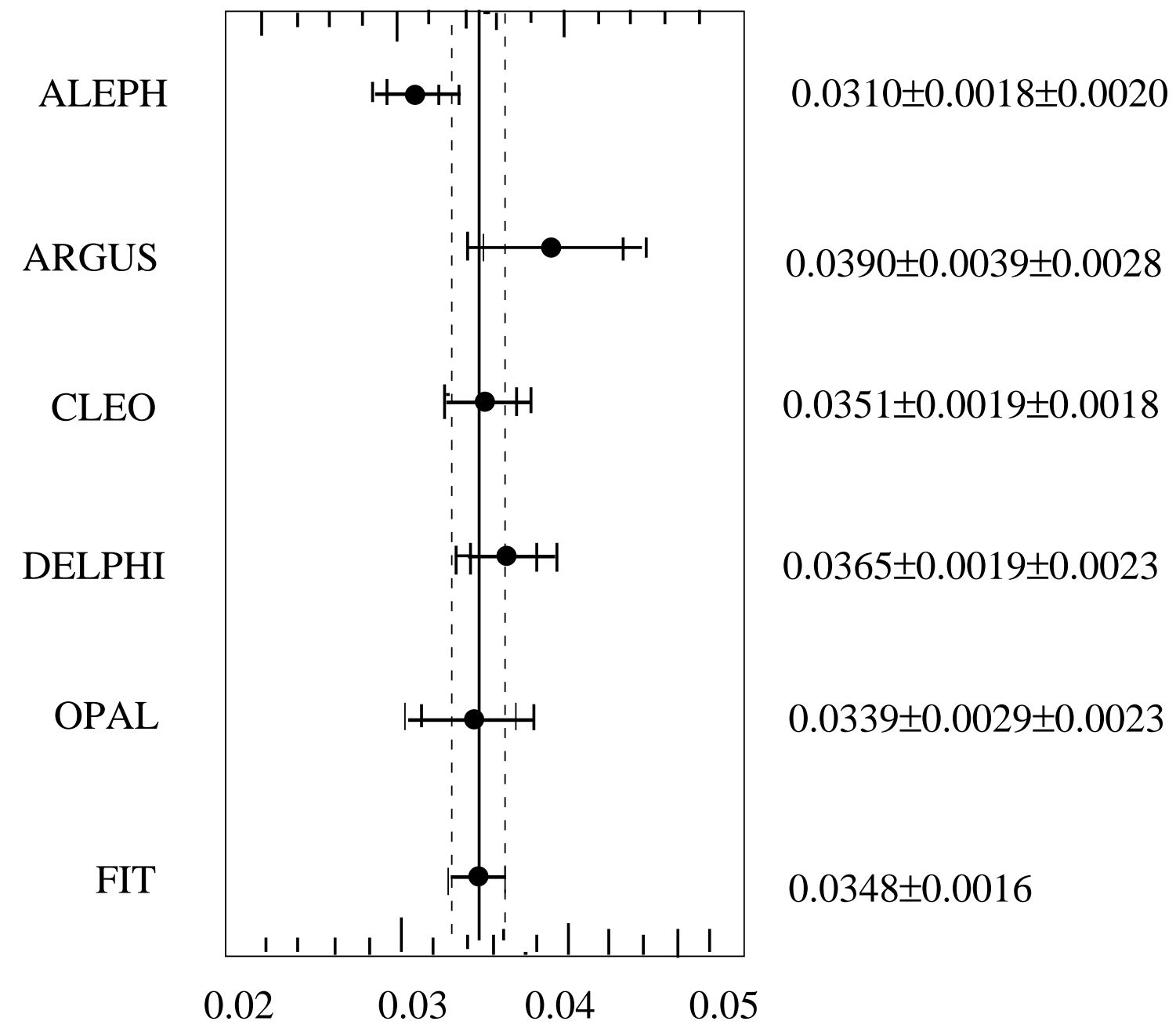

Figure 2: Determinations of $\left|V_{c b}\right| \mathcal{F}(w=1)$ from different experimental measurements. The average is also given. 
The scaling law in eq. (8), and others which can be derived for the form factors relevant in $B \rightarrow \rho l \nu_{l}$ or $B \rightarrow K^{*} \gamma$ decays, are valid in the heavy meson rest frame, for small values of the light meson three-momentum, i.e. for $\vec{p}_{\pi} \ll M_{P}$. Scaling laws at $q^{2} \sim 0$ can also be derived by computing the form factors using light-cone wave functions [33]. For example one finds $A_{1}\left(q^{2}=0\right) \sim M_{P}^{-3 / 2}$, where $A_{1}$ is the axial form factor entering in $B \rightarrow \rho l \nu_{l}$ decays. Unfortunately, corrections to the asymptotic scaling behaviour were found to be large both in lattice calculations [9, 32] and using QCD sum rules [33], cf. also the discussion on power corrections in sec. Q. A further constraint which can be used in theoretical predictions is that coming from the equality of some of the form factors in the helicity basis at $q^{2}=0$, e.g. $f^{+}\left(q^{2}=0\right)=f^{0}\left(q^{2}=0\right)$, and from analyticity [34. The above information is particularly useful for lattice calculations, where only a modest range of the physical $q^{2}$ is presently accessible.

Several theoretical methods have been used to predict the form factors for $B \rightarrow \pi$ and $B \rightarrow \rho$ decays. Besides the lattice calculations [9] and the QCD sum rules [33 mentioned above, several quark model calculations have also appeared in the literature. A complete list of theoretical calculations can be found in refs. [18, 26, 27]. It is interesting that, with the increasing experimental precision, it is possible to discriminate the "good" from the "bad" models by confronting the ratio of widths $\Gamma(B \rightarrow \rho) / \Gamma(B \rightarrow \pi)$, in which the unknown $\left|V_{u b}\right|$ cancels, to the theoretical predictions [18, 26, 27]. A compilation of results, obtained by the

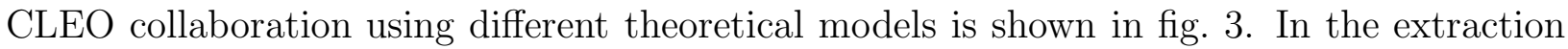
of $\left|V_{u b}\right|$, some models, the results of which are given in the figure above the horizontal line, have also been used in the Monte Carlo analysis of the data, in order to generate events to be confronted to the experimental measurements. The corresponding values for $\left|V_{u b}\right|$ are only given in these cases. The last value, denoted as "Endpoint method" refers, for comparison, to the result obtained from inclusive charmless decays, to be discussed in the following. From the figure, we see that the dominant error is the theoretical one. This is true in spite of the encouraging progresses made by the theoretical calculations in the last few years. These progresses are evident if one compares the results of fig. 3 to those available at the time of the 5th Symposium on Heavy Flavour Physics, held in Montreal in 1993 [35]. A further improvement of the present situation is likely to occur in the near future, particularly from lattice calculations.

\section{Exclusive non-leptonic decays}

In this section, I briefly discuss a few items connected to exclusive two-body non leptonic decays.

Factorization: Factorization is an interesting phenomenological idea which allows us to predict non-leptonic amplitudes in terms of semi-leptonic form factors and of vector and pseu- 


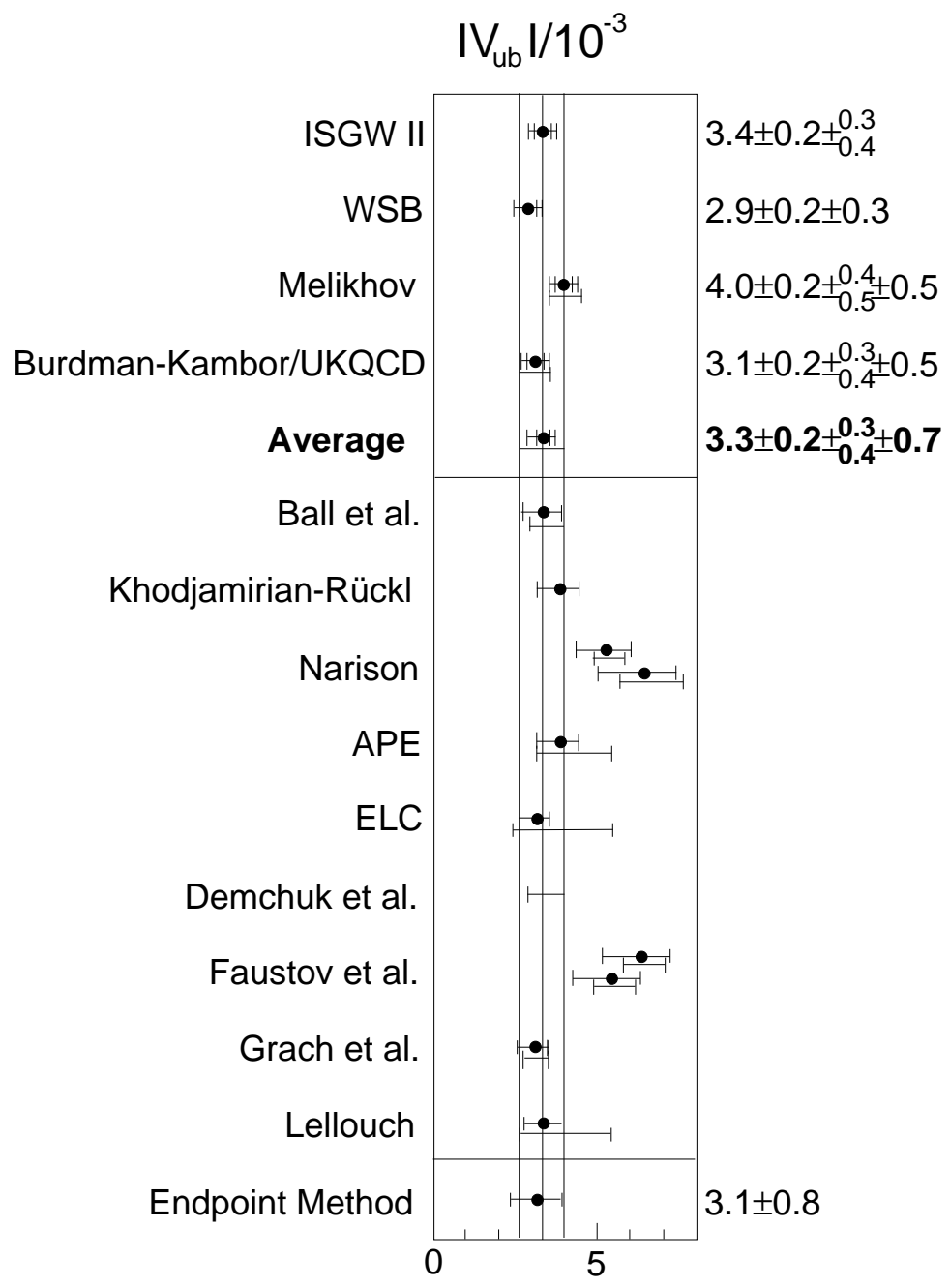

Figure 3: Experimental determinations of $\left|V_{u b}\right|$ from exclusive $B \rightarrow \pi$ and $B \rightarrow \rho$ semileptonic decays. The results depend on the theoretical model used in the experimental analysis. Only the models above the orizontal line have been used to extract $\left|V_{u b}\right|$ from the data. The value of $\left|V_{u b}\right|$ from inclusive decays is also given at the bottom of the figure. 
doscalar decay constants. In a way, it corresponds to an elaborated version of the vacuum saturation approximation. Factorization was originally used to estimate kaon decay amplitudes [36]. In the eighties, it was applied by Stech and collaborators in analyzing $D$-meson decays [37 and its explanation in terms of the $1 / N$ expansion, where $N$ is the number of colours, was subsequently proposed [38. More recently, it has been suggested that factorization holds in the heavy quark limit, for some energetic decay channels, because the ultrarelativistic light mesons in the final state do not have time to exchange gluons [39, 40]. In ref. 41] it was shown, however, that the demonstration of factorization given in [40 has some problems. It may be still true that factorization becomes valid in the infinite mass limit, but a convincing proof of this intuitive physical idea is still missing. Several observations are necessary at this point.

i) Factorization does not work for charm meson decays, where, moreover, large final-state interaction effects are measured (final-state interactions are zero if amplitudes factorize). This may be due either to a failure of factorization, or to the fact that, as observed above, the charm quark is not heavy enough. Indeed factorization, combined with the assumption that final-state interactions are dominated by nearby resonances, has been succesfully applied to phenomenological studies of charmed meson decays, for example in ref. 44].

ii) The effective Hamiltonian which controls weak charm decays has the form

$$
\mathcal{H}=\sum_{i} C_{i}\left(\frac{M_{W}}{\mu}\right) \hat{O}_{i}(\mu),
$$

where $\hat{O}_{i}(\mu)$ are composite operators renormalized at the scale $\mu$ and $C_{i}\left(M_{W} / \mu\right)$ the corresponding Wilson coefficients. The dependence on $\mu$ of the operators is compensated by the dependence on $\mu$ of the Wilson coefficients. The factorized matrix elements of the operators, however, are $\mu$-independent. Thus the final physical amplitude, in the factorization approximation, seems to depend on the renormalization scale. It may be true that ther exists a scale at which non-factorizable contributions are small, e.g. $\mu=m_{b}$ for $B$-decays, but a violation of factorization must be present to some extent.

iii) As recently shown in ref. [43] factorization and quark-hadron duality are incompatible. To reconcile duality and factorization a certain amount of non-factorizable contributions has to be allowed.

In conclusion, we know that non-factorizable contributions ought to be there, and that they are important for charm decays. The basic question is then to have a quantitative estimate of their size in $B$-decays. This problem has been studied in extensive experimental analyses of two-body exclusive decays [26] and it could be possibly accessible to lattice simulations in the near future [44], see below.

Non-leptonic decays on the lattice: There exist only quite old (and not very accurate) studies of heavy-quark two-body non-leptonic decays on the lattice, performed at $m_{Q} \sim m_{\text {charm }}$ 


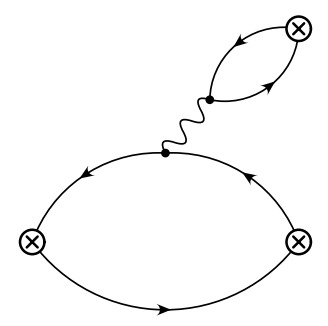

$\mathrm{DE}$

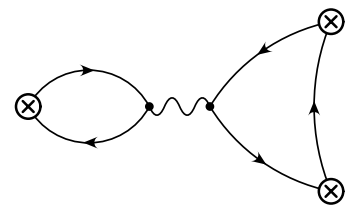

DA

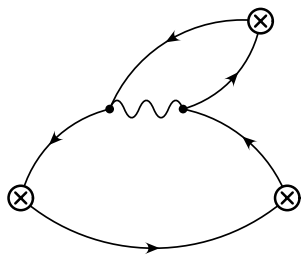

$\mathrm{CE}$

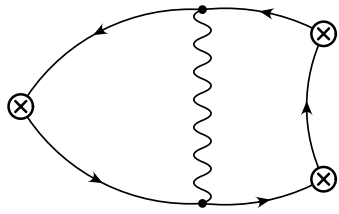

CA

Figure 4: Non-penguin Feynman diagrams for non-leptonic two-body decays.

45]. In these calculations only "non-penguin" amplitudes were considered, namely those corresponding to the following Feynman diagrams: disconnected emission (DE), denoted also as $T$ or $T^{\prime}$ in ref. [46]; the colour-suppressed connected (non-factorizable) emission (CE), denoted also as $C$ or $C^{\prime}$; the helicity suppressed disconnected annihilation (DA), denoted also as $A$ or $A^{\prime}$, and connected annihilation (CA), denoted also as $E$ or $E^{\prime}$. These diagrams are given in fig. $甘$.

In [45] it was found that the amplitude of the non-factorizable diagram CE was about one third of the leading contribution given by DE, as expected on the basis the $1 / N$ expansion, and that the amplitude of DA was indeed compatible with zero. The amplitude corresponding to CA, which is assumed to be negligible in some theoretical analyses [46], was found, however, to be as large as one half of that from DE. All the above results were unfortunately afflicted by large statistical and systematical errors, and have never been repeated since. The reason for this is that all lattice calculations of two-body non-leptonic amplitudes were abruptly interrupted when the Maiani-Testa no-go theorem was published [47. This theorem states the following. In numerical simulations on the lattice, it is unavoidable to make a Wick rotation to the Euclidean space-time, where $i n$ - and out-states are indistinguishable. From the standard study of the correlation functions one obtains then the average of the in- and out- amplitudes instead of the physical one, thus loosing all information about final 
state interactions f. For example, in the calculation of the decay of a $B$-meson into two light mesons $M_{1}$ and $M_{2}$, mediated by the weak Hamiltonian $\mathcal{H}^{W}$, one gets

$$
A^{W}=\frac{1}{2}\left({ }_{\text {out }}\left\langle M_{1} M_{2}\left|\mathcal{H}^{W}\right| B\right\rangle+{ }_{\text {in }}\left\langle M_{1} M_{2}\left|\mathcal{H}^{W}\right| B\right\rangle\right)
$$

instead of ${ }_{\text {out }}\left\langle M_{1} M_{2}\left|\mathcal{H}^{W}\right| B\right\rangle$ only. Moreover, at large time-distances, the correlation functions from which the amplitudes are usually extracted are dominated by the off-shell matrix element $\left\langle M_{1}\left(\vec{p}_{1}=0\right) M_{2}\left(\vec{p}_{2}=0\right)\left|\mathcal{H}^{W}\right| B\right\rangle$, instead of the physical amplitude. In general, the Maiani-Testa theorem prevents the calculation of time-like electro-magnetic form factors and strong and weak decays in two or more hadron final states. Its extension can be used to show that it also limits the possibility of computing inclusive cross-sections and decay rates [49]. A possible way out of this problem, under mild assumptions, although at the price of introducing some model dependence, has been recently suggested in ref. 444. We do not know at present if the proposal of ref. [44] will, in practice, succeed in extracting the physical amplitudes including the phases due to final state interactions. Feasibility studies are currently underway. If the proposal works, a new field of investigation (factorization, scaling laws, effects due to nearby resonances) will be open to lattice calculations.

Other interesting two-body amplitudes:

i) Penguin Pollution: in $B^{0} \rightarrow \pi^{+} \pi^{-}$decays, CP violating effects are given by the combined contribution of tree-level diagrams and penguin diagrams [50. In order to extract the relevant phases, it is the necessary to discriminate the two effects. Experimentally, this can be achieved by separating the term proportional to $\cos \Delta M_{B_{d}} t$ from that proportional to $\sin \Delta M_{B_{d}} t$ in the time-dependent asymmetry

$$
\mathcal{A}=\frac{N\left(B_{d}^{0} \rightarrow \pi^{+} \pi^{-}\right)(t)-N\left(\bar{B}_{d}^{0} \rightarrow \pi^{+} \pi^{-}\right)(t)}{N\left(B_{d}^{0} \rightarrow \pi^{+} \pi^{-}\right)(t)+N\left(\bar{B}_{d}^{0} \rightarrow \pi^{+} \pi^{-}\right)(t)} .
$$

A theoretical calculation of the tree-level and penguin contributions using the method of ref. [44], combined with the experimental analysis, would certainly help to reduce the systematic uncertanties in the final results.

ii) Isospin analysis: by combining the measured rates of the $B^{0} \rightarrow \pi^{+} \pi^{-}$and $B^{0} \rightarrow \pi^{0} \pi^{0}$ decays, one can remove the penguin contribution, by isolating the pure $\Delta I=3 / 2$ amplitude [50]. The difficulty of this method is due to the measurement of the decay rate into two neutral pions, which is expected to be colour suppressed. An alternative method, based on a combined study of $B^{0} \rightarrow \pi \pi, B^{0} \rightarrow \pi K$ and $B^{0} \rightarrow K \bar{K}$ decays, using approximate flavour $S U(3)$ symmetry has also been proposed. There is no space to discuss the details, and uncertainties, of this method, for which the reader can refer to [50]. I want only to add one observation, which may stimulate further investigation. In refs. [46, 50], it is usually

\footnotetext{
${ }^{5}$ In ref. [48], a similar problem was found in the context of QCD sum rules.
} 
assumed that the non-factorizable annihilation diagram CA is negligible, in contrast, but within large errors, and for smaller heavy quark masses, with the results of ref. [45], see above. A reappraisal of the lattice calculations of the non-leptonic amplitudes, on large lattices, and by using the theoretical improvements developed in the last few years 49] would certainly be very useful in this respect.

\section{Power corrections in the HQET}

In this section the problem of controlling power corrections in the heavy quark effective theory (HQET) is discussed. These corrections are necessary for an accurate evaluation of both inclusive and exclusive decays of hadrons containing a $b$-quark. The basic question is whether they can be computed with a theoretical error smaller than the corrections themselves [25].

To be specific, we start by considering the generic expression for the inclusive decay rate [51]-55.

$$
\Gamma\left(H_{b} \rightarrow X_{f}\right)=\frac{G_{F}^{2} m_{b}^{5}}{192 \pi^{3}}\left\{\left(1+\frac{\lambda_{1}+3 \lambda_{2}}{2 m_{b}^{2}}\right) C_{f}^{3}+\frac{6 \lambda_{2}}{m_{b}^{2}} C_{f}^{5}+\frac{16 \pi^{2} f_{B}^{2} M_{B}}{m_{b}^{3}} \sum_{i} C_{i}^{6} B_{i}^{6}+\ldots\right\},
$$

and the ratio of form factors in $B \rightarrow D^{*} l \nu_{l}$ decays 4$]$

$$
\frac{h^{V}\left(\omega=v \cdot v^{\prime}\right)}{h^{A}\left(\omega=v \cdot v^{\prime}\right)}=\frac{C^{V}(\omega)}{C^{A}(\omega)}\left(1+\frac{\bar{\Lambda}}{m_{c}} f(\omega)+\ldots\right) .
$$

In the above expressions $h^{V, A}$ are the relevant vector and axial vector form factors; $C_{f}^{3}, C_{f}^{5}$, $C_{i}^{6}, C^{V}(\omega)$ and $C^{A}(\omega)$ are short distance quantities that can be computed in perturbation theory; the quark mass $m_{b}$, the binding energy $\bar{\Lambda}$, the kinetic energy $\lambda_{1}$, the chromo-magnetic term $\lambda_{2}$ and the $B_{i}^{6}$ 's, that are related to the matrix elements of four-fermion operators, require instead a non-perturbative evaluation.

In some cases the non-perturbative parameters can be directly related, at the leading order in $1 / m_{Q}$, to a physical quantity. This is the case of the chromo-magnetic term which corresponds to the $B^{*}-B$ mass splitting, $\lambda_{2}=1 / 4\left(M_{B^{*}}^{2}-M_{B}^{2}\right)$. In general, however, the coefficients of the $1 / m_{Q}$ expansion, and the corresponding non-perturbative parameters, are plagued by renormalon ambiguities. This problem is present for example in the definition the binding energy $\bar{\Lambda}$ appearing in eq. (13) and in the matrix elements $B_{i}^{6}$ of the four fermion operators in eq. (12] [25], [56]-[58]. In these cases, the cancellation of the ambiguities occurs between the matrix elements of the higher dimensional operators and the Wilson coefficient functions $\left(C_{f}^{3}, C_{f}^{5}, \ldots\right)$. The latter have to be computed to sufficiently high order of perturbation theory for the cancellation to be under control. Let us imagine that we want to achieve an accuracy of $O\left(1 / m_{b}^{k}\right)$. A simple argument indicates that, in principle, the leading Wilson 
coefficient has to be computed at least to an order such that $\alpha_{s}\left(m_{b}\right)^{n} \sim\left(\Lambda_{Q C D} / m_{b}\right)^{k}$ [25, 59]. Since, in the calculations performed up to now, only the first few terms of the perturbation series are known, it is not possible to check that the remaining terms are negligible. By studying some simple examples, it has been shown that the knowledge of only a few terms is, in general, insufficient to control the power corrections [25]. Although the results rely on some approximations that were adopted in these examples, it is likely that the conclusions will remain valid in general, and that in most cases a further theoretical effort is needed.

Let us consider, for example, the unphysical parameter $\bar{\Lambda}=M_{H}-m_{\text {pole }}$ which is frequently used in phenomenological studies of $B$-physics. Results and bounds for $\bar{\Lambda}$ have been presented in the literature 600- 63]. In order for this to make any sense, $\bar{\Lambda}$ must be defined precisely in terms of some physical quantity, and its value will depend on this physical quantity and on the order of perturbation theory used to extract $\bar{\Lambda}$. In ref. [25] it has been shown that, even by using a consistent and renormalon-free definition of the binding energy, one expects that the value of $\bar{\Lambda}$ will change by several hundred $\mathrm{MeV}$, i.e. by an amount comparable to its value, as the order of perturbation theory is increased. The error on $\bar{\Lambda}$ can be seen as an error on the $\overline{\mathrm{MS}}$ mass of the $b$-quark $m_{b}^{\overline{\mathrm{MS}}}\left(m_{b}^{\overline{\mathrm{MS}}}\right)$ as derived from the HQET [64]

$$
m_{b}^{\overline{\mathrm{MS}}}\left(m_{b}^{\overline{\mathrm{MS}}}\right)=4.17 \pm 0.05 \pm 0.20 \mathrm{GeV},
$$

where the last (and larger) error comes from an estimate of the uncertainty due to higher order corrections in perturbation theory.

Similar problems are encountered in the case of $\lambda_{1}$. One example is given by the zero recoil sum rules [67, 68]. In this case, in order to derive bounds on the relevant form factors, and on the heavy-quark kinetic energy parameter $\lambda_{1}$, a physical cut-off $\Delta$ is introduced, to suppress the contribution of states with excitation energies greater than $\Delta$. The difficulty in achieving an accurate determination or bound with a physical cut-off is due to the higher order perturbative corrections and only by arriving to an order such that $\alpha_{s}^{n} \leq O\left(\lambda_{1} / \Delta^{2}\right)$ it will be possible to obtain significant results. A connected example is provided by the different theoretical determinations of $\lambda_{1}$, which are given in table 2. Some of them are not compatible, if we accept as realistic the errors quoted in the original papers. I believe that the differences are due to higher order perturbative corrections, which have not been computed, or estimated, yet.

\section{Semi-leptonic inclusive decays}

The idea that inclusive decay rates of heavy quarks can be computed in the parton model is quite old and was used, for example, in ref. 73 to predict charm hadron lifetimes. To account for bound state effects, the partonic calculation was successively improved by the introduction of a phenomenological model, called the "spectator model" [74, 75], which has 


\begin{tabular}{||c|c|c||}
\hline \hline \multicolumn{3}{|c|}{ Theoretical estimates of $\lambda_{1}$} \\
\hline \hline Reference & Method & $-\lambda_{1}\left(\mathrm{GeV}^{2}\right)$ \\
\hline \hline Eletsky and Shuryak [65] & QCD sum rules & $0.18 \pm 0.16$ \\
Ball and Braun [66] & QCD sum rules & $0.52 \pm 0.12$ \\
Bigi et al. [67] & ZR sum rules & $\geq 0.36$ \\
Kapustin et al. [68] & ZR sum rules $+O\left(\alpha_{s}\right)+O\left(\alpha_{s}^{2} \beta_{0}\right)$ & -- \\
Ligeti and Nir [69] & Experiment & $\leq 0.63$ if $\bar{\Lambda} \geq 240 \mathrm{MeV}$ \\
& Experiment & $\leq 0.10$ if $\bar{\Lambda} \geq 500 \mathrm{MeV}$ \\
Giménez et al. [70] & Lattice & $-0.09 \pm 0.14$ \\
Chernyak [71] & Experiment & $0.14 \pm 0.03$ \\
Gremm et al. [63] & Experiment & $0.19 \pm 0.10$ \\
Neubert [72] & Virial Theorem & $0.10 \pm 0.05$ \\
\hline \hline
\end{tabular}

Table 2: Some of the values of $\lambda_{1}$ obtained in different theoretical analyses. "Experiment" denotes the extraction of $\lambda_{1}$ from the experimental data, for example the charged-lepton spectrum distribution in semi-leptonic B-meson decays. "ZR sum rules" denotes the zero recoil sum rules.

been and continues to be extensively used to extract $\left|V_{c b}\right|$ and $\left|V_{u b}\right|$ from inclusive semileptonic decays. A noble theoretical framework for the spectator model was then provided by the operator product expansion applied to inclusive decays of heavy quarks [51] [55]. The general result is summarized in eq. (12), which holds for both inclusive semi-leptonic and non-leptonic decays. The first term on the r.h.s. of eq. (12) is the parton model result; corrections of $O\left(1 / m_{b}^{2}\right)$ are due to the kinetic energy $-\lambda_{1}$ and the spin interaction $\lambda_{2}$ of the heavy quark in the hadron [; the four-fermion operator contributions to the $1 / \mathrm{m}_{b}^{3}$ correction, written in terms of the $B_{i}^{6}$ parameters, correspond, in the old language, to $\mathrm{W}$-exchange and interference higher-twist effects. These effects were considered in order to explain the lifetime differences among the different charmed hadrons [76].

In modern language, the use of the parton model to compute inclusive decays relies on the assumption of "quark-hadron" duality: in semi-leptonic decays, where the integration over the lepton and neutrino momenta corresponds to an integration over the invariant mass of the recoiling hadronic system, we have global duality, whereas in non-leptonic decays, where the hadronic mass is fixed to be equal to the heavy hadron mass, we speak of local duality. Duality is assumed, but cannot be proven in QCD. We know that it provides a good description of several physical processes such as inclusive deep inelastic scattering, inclusive $e^{+} e^{-}$annihilation into hadrons, Drell-Yan processes, etc. The assumption of global duality seems also to work for semi-leptonic (and radiative) decays. This means that we are able to

\footnotetext{
${ }^{6}$ Up to a redefinition of the quark mass, there is indeed a relation between the Fermi momentum of the heavy quark in the model of ref. [75] and $\lambda_{1}$.
} 
predict the inclusive $\Gamma_{S L}\left(b \rightarrow X_{c} l \nu_{l}\right), \Gamma_{S L}\left(b \rightarrow X_{u} l \nu_{l}\right)$ and $\Gamma_{S L}(b \rightarrow s \gamma)$ rates. This is not the case for local duality since there is strong experimental evidence that we are unable to explain $B$-hadron lifetimes in the framework of the $1 / m_{b}$ expansion, see sec. 7 .

The situation for $\Gamma_{S L}\left(b \rightarrow X_{c} l \nu_{l}\right)$ is substantially unchanged since last year. For this reason, I direct the reader to ref. [7] for a discussion of the theoretical uncertainties in the calculation of this quantity. Here I limit myself giving the upgraded world average values of $\left|V_{c b}\right|$ from inclusive decays

$$
\begin{aligned}
\left|V_{c b}\right|^{\Upsilon(4 s)} & =0.0391 \pm 0.0007 \pm 0.0030 \\
\left|V_{c b}\right|^{L E P} & =0.0412 \pm 0.0004 \pm 0.0030
\end{aligned}
$$

where the results from low energy and LEP energies have been given separated. The reason is that the different central values are induced mostly by the differences in the semi-leptonic branching ratio measured in the two cases [18. Notice the excellent agreement between these results and the number given in eq. (7).

The above results make us confident that we are able to make accurate predictions for $\Gamma_{S L}\left(b \rightarrow X_{c} l \nu_{l}\right)$. A related problem is whether we are also able to predict the semi-leptonic branching ratio $B_{S L}$, and the average number of charmed hadrons $n_{c}$. Since the former is given by the ratio of $\Gamma_{S L}\left(b \rightarrow X_{c} l \nu_{l}\right)$ to the total width, the problem is whether we are able to predict inclusive non-leptonic widths. Recently, there was a lot of debate about whether the theoretical value of $B_{S L}$ is in agreement, or in disagreement, with the experimental measurements [55, 77, 78 and, in the second case, several explanations, within and beyond the standard model, have been proposed [79]-82]. Since there are important differences between low energy and LEP results [18]

$$
\begin{array}{rll}
B_{S L}^{\Upsilon(2 s)}=10.49 \pm 0.17 \pm 0.43, & n_{c}^{\Upsilon(2 s)}=1.12 \pm 0.05 \\
B_{S L}^{L E P}=10.95 \pm 0.13 \pm 0.29, & n_{c}^{\Upsilon(2 s)}=1.22 \pm 0.08
\end{array}
$$

it is probably premature to draw any conclusion at this stage.

$\left|V_{u b}\right|$ could be extracted, in principle, from the total semi-leptonic width in the same way as $\left|V_{c b}\right|$. In practice, however, only the endpoint spectrum is used, in order to eliminate the background due to $b \rightarrow c l \nu_{l}$ decays. The cut on the lepton energy $E_{l}$ is fixed by the requirement that the recoiling hadronic system $X$ has an invariant mass $M_{X} \leq M_{D}$, so as to inhibit charm decays. In this region, however, we run into troubles with the operator product expansion. Schematically, we can write

$$
\frac{d \Gamma\left(b \rightarrow u l \nu_{l}\right)}{d E_{l}}=\sum_{n} \int_{y_{\min }\left(E_{l}\right)}^{y_{\max }\left(E_{l}\right)} d y\left(\frac{\Lambda_{Q C D}}{m_{b}(1-y)}\right)^{n} \Gamma_{n}(y)+\ldots,
$$

where ... represent terms which are suppressed as powers of $m_{b}$. Only the first few terms in the expansion above are known because they can be related to $\lambda_{1}, \lambda_{2}$, etc. The higher order 
terms depend on matrix elements of higher dimensional local operators, which can at most be estimated within a specific phenomenological model.

Near the end point, $y_{\min } \sim 1-M_{X}^{2} / m_{b}^{2}\left(y_{\max }\right.$ is always of $\left.O(1)\right)$ so that when $M_{X} \sim$ $\sqrt{\Lambda_{Q C D} M_{B}}$, all the terms in the expansion (17) become equally important. At even larger values of $E_{l}, M_{X} \sim \Lambda_{Q C D}$. In this case we are in the elastic region (corresponding to $B \rightarrow \rho$, $B \rightarrow \pi$, etc.) and the decay cannot be analyzed with operator product expansion. Unfor-

tunately, $M_{X} \sim \sqrt{\Lambda_{Q C D} M_{B}}$ is about $1.2-1.3 \mathrm{GeV}$, a value very close to the kinematical cut which is currently used in the experiments to extract $\left|V_{u b}\right|$. This implies that the extraction of $\left|V_{u b}\right|$ is still subject to an intrinsic model dependence, which can be reduced either by enlarging the region of $E_{l}$ from where $\left|V_{u b}\right|$ is extracted, at the price of increasing the charm background, or by a sound theoretical prediction of the "wave" function [52, 54] of the heavy quark.

\section{Non-leptonic inclusive decays}

In this section it will be shown that it is very unlikely that higher order corrections in $1 / m_{b}$ can explain the discrepancy between the theoretical predictions for the $B$ to $\Lambda_{b}$ lifetime ratio $R=\tau_{B} / \tau_{\Lambda_{b}}$.

For $R$, the dependence on the fifth power of the quark mass, as well as all renormalon ambiguities up to order $1 / m_{b}^{3}$, cancel out. We thus expect $R$ to be one of the soundest predictions of HQET. The discrepancy between theory and experimental data is evidence for the failure of local duality in the calculation of the non-leptonic (NL) widths of heavy hadrons.

To order $1 / m_{b}^{2}$ in the heavy quark expansion, the lifetime ratio $R$ is given by

$$
R=1+\frac{\lambda_{1}(B)-\lambda_{1}\left(\Lambda_{b}\right)}{2 m_{b}^{2}}+C_{G} \frac{\lambda_{2}(B)-\lambda_{2}\left(\Lambda_{b}\right)}{m_{b}^{2}}+O\left(1 / m_{b}^{3}\right),
$$

where the difference of kinetic energies $\lambda_{1}(B)-\lambda_{1}\left(\Lambda_{b}\right)=0.01 \pm 0.03 \mathrm{GeV}^{2}$ can be obtained from the spectrum of the heavy hadrons; $\lambda_{2}\left(\Lambda_{b}\right)=0 ; \lambda_{2}(B)=\left(M_{B^{*}}^{2}-M_{B}^{2}\right) / 4=0.12 \mathrm{GeV}^{2}$ and $C_{G} / 3 \sim 1.1$. Using the numerical values given above one obtains $R=1.02+O\left(1 / m_{b}^{3}\right)$, incompatible with the observed ratio of lifetimes $R=1.27 \pm 0.05$. Within the standard HQET, in order to explain the large deviation from unity for this ratio it is necessary the the matrix elements of the four-quark operators entering in the $1 / m_{b}^{3}$ corrections (and in particular those relative to the $\Lambda_{b}$ ) be much larger than those estimated in quark models, and more recently with QCD sum rules [55, 80]. In my opinion, this remains an open, but very unlikely possibility. An alternative explanation is that for NL widths there is evidence, contrary to the orthodox expectations, of corrections of $O\left(1 / m_{b}\right)$. These corrections are well 
described by the simple ansatz that replaces the quark mass with the decaying hadron mass in the $m_{Q}^{5}$ factor in front of the NL width [8, 79]

$$
\Gamma\left(H_{b} \rightarrow X_{f}\right)=\left.\frac{G_{F}^{2} m_{b}^{5}}{192 \pi^{3}}\right|_{H Q E T}+O\left(1 / m_{b}^{2}\right) \rightarrow \frac{G_{F}^{2} M_{H}^{5}}{192 \pi^{3}}+O\left(1 / M_{H}^{2}\right) .
$$

This replacement provides a much better description of the NL widths. In ref. [79], it was shown that, for beauty, both the problems of the SL branching ratio and of the difference in the lifetimes of the $\Lambda_{b}$ baryon and the $B$ mesons are quantitatively solved. For charm a much better fit to the seven known lifetimes is obtained in terms of four parameters of reasonable size: one lifetime, one interference contribution for $D^{+}$, one for $\Xi^{+}$and a smaller W-exchange term for $D^{s}$. I will now give more details on the analysis of ref. [79].

Given the agreement between the value of $\left|V_{c b}\right|$ extracted from inclusive $b \rightarrow c$ and exclusive $B \rightarrow D^{*}$ semi-leptonic (SL) decays and the approximate equality of the SL widths for charmed hadrons (within large uncertainties and with a rather limited number of cases where measurements exist), it is assumed that the HQET is able to predict the SL widths $\Gamma_{S L}$ of the heavy hadrons. Under this assumption, and using the ansatz (19), one gets

$$
R=\left(\frac{m_{\Lambda_{b}}}{m_{B}}\right)^{5}\left[1-2.24 B_{S L}(B)\right]+2.24 B_{S L}(B)+O\left(1 / M_{H}^{2}\right),
$$

where the factor 2.24 arises from taking the electron, the muon and the tau SL modes in the ratio 1:1:0.24 [83], and the difference in the SL rates of the $\Lambda_{b}$ and $B$ has been neglected. From $M_{\Lambda_{b}}=5623 \pm 6 \mathrm{MeV}$ [84] and $M_{B}=5279 \pm 2 \mathrm{MeV}$ [85], by using $B_{S L}(B)=10.77 \pm 0.43 \%$, one finds \

$$
\frac{\tau_{B}}{\tau_{\Lambda_{b}}}=1.29 \pm 0.05
$$

in perfect agreement with the experimental result.

So far this agreement can be considered to be a numerical accident, lacking theoretical explanation. For this reason, it is quite interesting to consider what happens in the case of charmed particles for which seven lifetimes have been measured, see table 3. At lowest order in the $1 / m_{Q}$ expansion, a much better agreement with the experimental results for the lifetimes is obtained by replacing the heavy-quark mass by the hadron masses as in eq. (19). We neglect at this stage any other mass correction and write $\Gamma_{N L}\left(M_{H}\right)=\Gamma_{t o t}\left(M_{H}\right)-2 \Gamma_{S L}$, where for $\Gamma_{S L}$ a universal value chosen as the average of the experimental values for $D^{+}, D^{0}$ and $\Lambda_{c}$, or $\Gamma_{S L}=(0.174 \pm 0.015) \mathrm{ps}^{-1}$ is used [85]. The dependence on the hadron mass of $\Gamma_{N L}\left(M_{H}\right)$ will be taken according to $\Gamma_{N L}\left(M_{H}\right)=\left(M_{H} / M_{0}\right)^{n} \Gamma_{N L}\left(m_{0}\right)$ with $\mathrm{n}=5$, where $M_{0}$ is around the average mass of the relevant hadron. We then have

$$
\Gamma_{t o t}\left(M_{H}\right)=\tau^{-1}\left(M_{H}\right)=\tau^{-1}\left(M_{0}\right)\left(\frac{M_{H}}{M_{0}}\right)^{n}+2 \Gamma_{S L}\left(1-\left(\frac{M_{H}}{M_{0}}\right)^{n}\right)
$$




\begin{tabular}{||c|c|c|c|c|}
\hline Hadron & Mass $\left(\mathrm{MeV} / c^{2}\right)$ & $\tau(\mathrm{ps})$ & $B_{S L}(\%)$ & $\Gamma_{S L}=B_{S L} / \tau\left(\mathrm{ps}^{-1}\right)$ \\
\hline$D^{ \pm}$ & $1869.4 \pm 0.4$ & $1.057 \pm 0.015$ & $17.2 \pm 1.9$ & $16.3 \pm 1.8$ \\
$D^{0}$ & $1864.6 \pm 0.5$ & $0.415 \pm 0.004$ & $8.1 \pm 1.1$ & $19.5 \pm 2.6$ \\
$D_{s}$ & $1968.5 \pm 0.7$ & $0.467 \pm 0.017$ & & \\
$\Lambda_{c}^{0}$ & $2285.1 \pm 0.6$ & $0.200 \pm 0.011$ & $4.5 \pm 1.7$ & $22.5 \pm 8.5$ \\
$\Xi_{c}^{0}$ & $2470.3 \pm 1.8$ & $0.098 \pm 0.019$ & & \\
$\Xi_{c}^{ \pm}$ & $2465.1 \pm 1.6$ & $0.350 \pm 0.055$ & & \\
$\Omega_{c}^{0}$ & $2704 \pm 4$ & $0.055 \pm 0.023$ & & \\
\hline
\end{tabular}

Table 3: Properties of charmed mesons and baryons; the $\Omega^{0}$ values are an average of the data quoted in ref. 80].

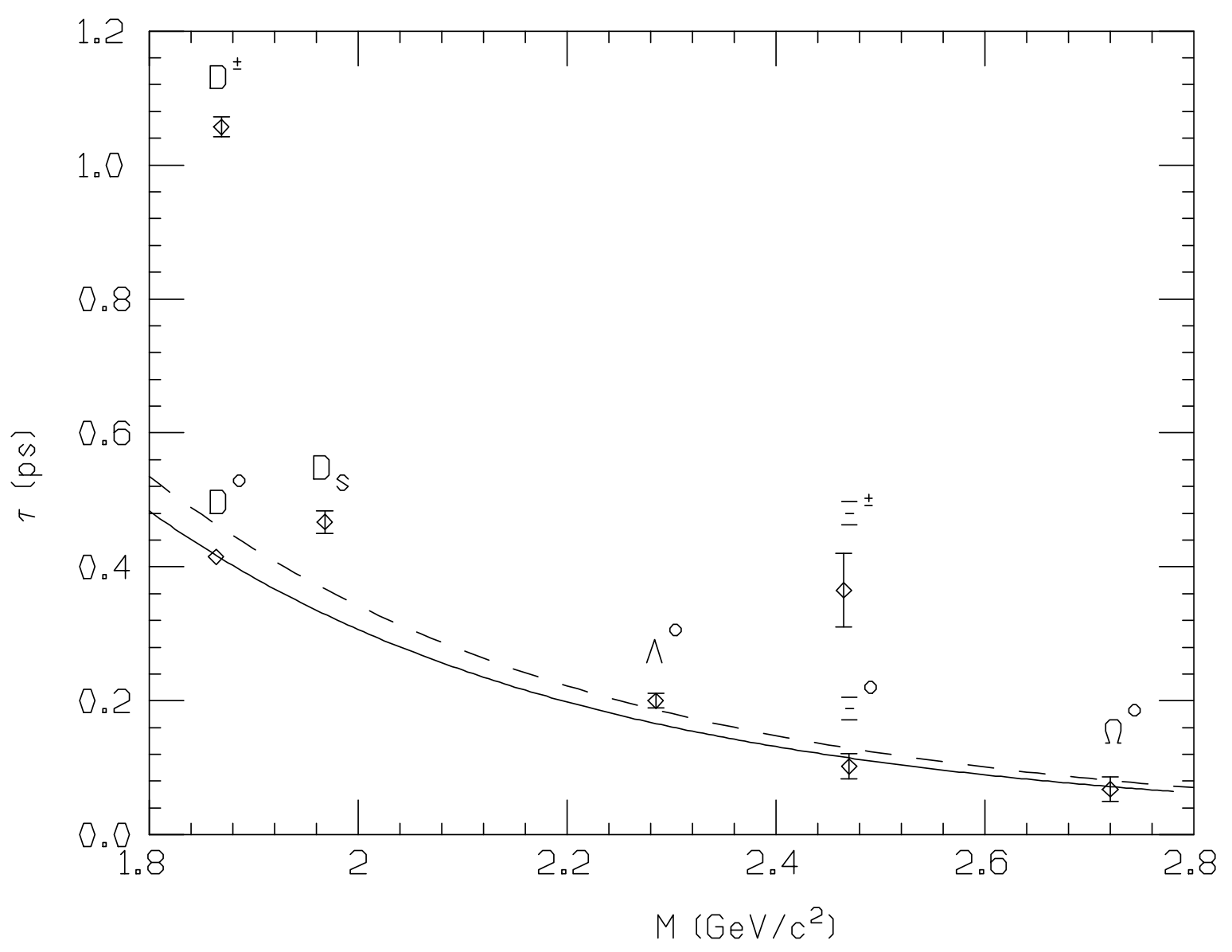

Figure 5: Lifetime vs. mass for charmed particles. The dashed line is the best fit described in the text for all seven points assuming proportionality of the $N L$ widths to $M_{H}^{5}$, $M_{H}$ being the hadron mass. The solid line is the best fit restricted to only the $D^{0}, \Lambda_{c}, \Xi^{0}$ and $\Omega_{c}$ lifetimes with the same assumptions as before. 
Let us fix $n=5, M_{0}=2.3 \mathrm{GeV}$ and $\Gamma_{S L}=0.174 \mathrm{ps}^{-1}$ and fit all seven known lifetimes in terms of $\tau\left(M_{0}\right) \cdot \tau\left(m_{0}\right)=0.181 \mathrm{ps}$ is then obtained. The corresponding fit is shown in fig. 5 (dashed curve). We see that four out of seven lifetimes are in very good agreement with the fitted curve. The lifetimes of $D^{+}$, of $\Xi^{+}$and, to a lesser extent, of $D_{s}$ are clearly out. The discrepancies for $D^{+}$and $\Xi^{+}$can be attributed to the interference effect [87]. Note that $D^{+}$ is the only meson that can have interference at the Cabibbo allowed level and $\Xi^{+}$is the only baryon that can have double interference, in the sense that $\Xi^{+}=$cus and both $u$ and $s$ can interfere with the corresponding quarks from $c \rightarrow s u \bar{d}$. For $D_{s}$ the observed smaller difference is attributed to the possibility of W-exchange [87]. All of these effects are of order $f_{D}^{2} / m_{c}^{2}$ or $1 / m_{c}^{3}$. The solid line in fig. 5 has been obtained from a modified fit where only the $D^{0}, \Lambda_{c}, \Xi^{0}$ and $\Omega_{c}$ lifetimes have been considered. In this case, $\tau\left(M_{0}\right)=0.161 \mathrm{ps}$, with the respectable value of the $\chi^{2} /$ d.o.f. given by $\sim 3.5$, is obtained. For comparison, the fit of the quark mass to constant lifetimes results in a $\chi^{2} /$ d.o.f. $\sim 250$. Finally, for the same four lifetimes, one fits the power $n$ in eq. (22), keeping fixed the value of $M_{0}$ and $\tau\left(M_{0}\right)$ at the observed values for the $D^{0}$ meson. In this way one may check whether the best power for $n$ is close to 5 . $n=4.5 \pm 0.5$ is found, where the error arises from the experimental errors on the lifetimes. Moreover, if we write for $D^{+}, \Xi^{+}$and $D_{s}$ the expression $\tau^{-1}=\tau^{-1}\left(M_{H}\right)\left[1-\left(\frac{\mu}{M_{H}}\right)^{3}\right]$, where $\tau$ is the experimental number given in table 3 and $\tau\left(M_{H}\right)$ is taken from the previous fit to the four remaining lifetimes (with $\mathrm{n}=5$ ) $, \mu=1.6,2.2$ and $1.3 \mathrm{GeV}$ are obtained for $D^{+}, \Xi^{+}$ and $D_{s}$, respectively. We see that the resulting values of this correction are large, as it is obvious from fig. 5, but not unreasonable.

The conclusion is that a number of experimental facts show that $\Gamma_{N L}$ for charm and beauty decay approximately scale with the fifth power of hadron masses (apart from corrections of order $1 / m_{Q}^{2}$ or smaller) rather than having $O\left(1 / m_{Q}^{2}\right)$ corrections to the universal behaviour predicted by the HQET. Since $M_{H_{b}}^{5}=\left(m_{b}+\bar{\Lambda}_{H_{b}}\right)^{5}=m_{b}^{5}\left(1+5 \Lambda_{H_{b}} / m_{b}\right)+\ldots$, this implies that the corrections are of $O\left(1 / m_{b}\right)$. Since the validity of the operator expansion in the timelike region, in the vicinity of the physical cut, is not at all guaranteed [88, 89, the failure of the short distance approach can be attributed to a violation of the local duality property that has to be assumed for NL widths. This is to be compared to the case of the SL widths for which the predictions of the HQET are not inconsistent with the data. The experimental evidence for NL widths calls for a reexamination of the underlying theoretical framework. An attempt in this direction can be found in ref. [81]. More extended and accurate measurements of the SL widths of each hadron separately are also needed in order to assess the validity of the heavy quark expansion in semi-leptonic decays.

\footnotetext{
${ }^{7}$ The same numbers as in ref. [79] are used here since the analysis has not been repeated since.
} 


\section{Conclusion and outlook}

Beauty hadrons provide an extraordinary laboratory to test the Standard Model, to look for hint of new physics and to understand QCD dynamics. For this reason an exceptional experimental and theoretical effort has been invested in it. In this review, I have tried to point out those dynamical aspects which we ought to understand and control at a quantitative level in order to extract the relevant information on some fundamental parameters, such as the mass of the $b$ quark, the relevant CKM matrix elements and CP violation.

Much theoretical progress, and improvement in understanding, has occured this year in the framework of the HQET and of the calculation of inclusive rates. Still a lot of work remains to be done, particularly for those quantities where the theoretical uncertainties are larger than the experimental errors. Progress can be expected quite soon for the slope and the curvature of the form factors in $B \rightarrow D^{*} l \nu_{l}$ decays, in the understanding of factorization and in the calculation of non-leptonic two-body decay amplitudes. It appears more difficult to reduce the error in the normalization of the form factor at zero recoil for $B \rightarrow D^{*} l \nu_{l}$ and to eliminate the model dependence in the extraction of $\left|V_{u b}\right|$ from inclusive semi-leptonic decays. A real puzzle, which demands a deep reappraisal of current ideas, and prejudices, remains that of the $\Lambda_{b}$ lifetime, which seems to indicate a failure of local duality in inclusive non-leptonic decays.

\section{Acknowlegments}

I thank M. Artuso, A.J. Buras, J. Flynn, M. Gronau, A. Masiero, M. Neubert, C.T. Sachrajda, S. Sharpe, and N. Uraltsev for informative, interesting, and intense discussions. I acknowledge the partial support by M.U.R.S.T. and by the EC contract CHRX-CT92-0051.

\section{References}

[1] M. Lindner, invited talk at Workshop on Electroweak Symmetry Breaking, Budapest, Hungary, 11-13 Jul 1994, TUM-HEP-214-94; HD-THEP-93-19, hep-ph/9306281 and refs. therein.

[2] C. Kounnas, I. Paval, G. Ridolfi and F. Zwirner, CERN-TH-95-11, hep-ph/9502318 and refs. therein.

[3] P. Ramond, R.G. Roberts and G.G. Ross, Nucl. Phys. B406 (1993) 19 and refs. therein.

[4] M. Neubert, Phys. Rep. 245 (1994) 259. 
[5] A. Ali and D. London, DESY-96-140, hep-ph/9607392.

[6] A.J. Buras, Nucl. Instrum. Meth. A368 (1995) 1; rapporteur talk given at the XXVIII International Conference on High Energy Physics, 25-31 July 1996, Warsaw (Poland), to appear in the Proceedings.

[7] M. Neubert, CERN-TH/96-55, hep-ph/9604412, and refs. therein.

[8] G. Martinelli, talk given at 6th International Symposium on Heavy Flavor Physics, Pisa, Italy, 6-9 Jun 1995, to appear in the Proceedings.

[9] J. Flynn, rapporteur talk given at Latt96, 14th International Symposium on Lattice Field Theory, St. Louis, 4-8 June 1996, to appear in the Proceedings; rapporteur talk given at the XXVIII International Conference on High Energy Physics, 25-31 July 1996, Warsaw (Poland), to appear in the Proceedings and refs. therein.

[10] C.T. Sachrajda at this workshop.

[11] M. J. Booth, Phys. Rev. D51 (1995) 2338; S. R. Sharpe and Y. Zhang, Phys. Rev. D53 (1996) 5125.

[12] A. Buras, M. Jamin and P.H. Weisz, Nucl. Phys. B347 (1990) 491.

[13] V. Giménez and G. Martinelli, ROME prep. 96/1153 and refs. therein.

[14] S. Narison, talk presented at the Cracow Epiphany Conference on Heavy Quarks, Montpellier prep. no. PM 95/05, hep-ph/9503234; see also [0].

[15] M. Ciuchini, E. Franco and G. Martinelli, unpublished.

[16] M. Lusignoli, L. Maiani, G. Martinelli and L. Reina, Nucl. Phys. B369 (1992) 139; for an update see for example M. Ciuchini, E. Franco, G. Martinelli, L. Reina and L. Silvestrini, Z. Phys. C68 (1995) 239; A.J. Buras, M. Jamin, and M.E. Lautenbacher, MPI-PH-96-57, hep-ph/9608365; see also [0] and refs. therein.

[17] L. Maiani, Nucl. Phys. B (Proc. Suppl.) 9 (1989) 193 and refs. therein.

[18] J. Richman, rapporteur talk given at the XXVIII International Conference on High Energy Physics, 25-31 July 1996, Warsaw (Poland), to appear in the Proceedings.

[19] M.E. Luke, Phys. Lett. B252 (1990) 447.

[20] A. Czarnecki, Phys. Rev. Lett. 76 (1996) 4124.

[21] A.F. Falk and M. Neubert, Phys. Rev. D47 (1993) 2965 and 2982; T. Mannel, Phys. Rev. D50 (1994) 428. 
[22] M.A. Shifman, N.G. Uraltsev and A. Vainshtein, Phys. Rev. D51 (1995) 2217.

[23] N.G. Uraltsev at this workshop.

[24] M. Neubert, Phys. Lett. B338 (1994) 84.

[25] G. Martinelli and C.T. Sachrajda, Phys. Lett. B354 (1995) 423; CERN-TH-96-117, hep-ph/9605336, to appear in Nucl. Phys. B

[26] M. Artuso at this workshop.

[27] L. Gibbons, rapporteur talk given at the XXVIII International Conference on High Energy Physics, 25-31 July 1996, Warsaw (Poland), to appear in the Proceedings.

[28] I. Caprini and M. Neubert, Phys. Lett. B380 (1996) 376.

[29] U. Aglietti, Phys. Lett. B301 (1993) 393; U. Aglietti, G. Martinelli and C.T. Sachrajda, Phys. Lett. B324 (1994) 85.

[30] UKQCD collaboration, L. Lellouch et al., Nucl. Phys. B444 (1995) 401.

[31] N. Isgur and M.B. Wise, Phys. Rev. D42 (1990) 2388.

[32] The APE collaboration, As. Abada et al., published in ICHEP94, (QCD161:H51:1994) p.713; Phys. Lett. B365 (1996) 275.

[33] A. Ali and V.M. Braun, Z. Phys. C63 (1994); P. Ball and V.M. Braun, Phys. Rev. D54 (1996) 2182.

[34] C. Boyd, B. Grinstein, R.F. Lebed, presented at 6th International Symposium on Heavy Flavor Physics, Pisa, Italy, 6-9 Jun 1995, to appear in the Proceedings, hep-ph/9508242; L. Lellouch, talk given at High-energy Physics International Euroconference on Quantum Chromodynamics (QCD 96), Montpellier, France, 4-12 Jul 1996, hep-ph/9609484 and refs. therein; see also [9].

[35] Proceedings of the 5th International Symposium on Heavy Flavor Physics, , Montreal, 6-10 July 1993, Eds. Frontiéres.

[36] M.A. Shifman, A.I. Vainshtein and V.J. Zakharov, Nucl. Phys. B120 (1977) 316; Sov. Phys, JEPT 45 (1977) 670.

[37] M. Bauer, B. Stech and M. Wirbel, Z. Phys. C34 (1987) 103.

[38] N. Deshpande, M. Gronau and D. Sutherland, Phys. Lett. B90 (1980) 431; A.J. Buras, J.M. Gérard and R. Rücl, Nucl. Phys. B268 (1986) 16. 
[39] J. Bjorken, Nucl. Phys. B (Proc. Suppl.) 11 (1989) 325.

[40] M. Duncan and B. Gristein, Phys. Lett. B255 (1991) 583.

[41] U. Aglietti, Phys. Lett. B292 (1992) 424.

[42] F. Buccella et al., Phys. Rev. D51 (1995) 3478.

[43] A. Le Yaouanc, L. Oliver, O. Pène and J.-C. Raynal, Phys. Rev. D52 (1995) 2813.

[44] M. Ciuchini, E. Franco, G. Martinelli, and L. Silvestrini, Phys. Lett. B380 (1996) 353.

[45] C. Bernard, J. Simone and A. Soni, Nucl. Phys. B (Proc. Suppl.) 17 (1990) 504; As. Abada et al., Nucl. Phys. B (Proc. Suppl.) 17 (1990) 518.

[46] M. Gronau, O.F. Hernandéz, D. London and J.L. Rosner, Phys. Rev. D50 (1994) 4529; D52 (1995) 6374; Phys. Lett. B333 (1994) 500; M. Gronau, D. London and J.L. Rosner, Phys. Rev. Lett. 73 (1994) 21.

[47] L. Maiani and M. Testa, Phys. Lett. B245 (1990) 585.

[48] B.Yu. Blok and M.A. Shifman, Sov. J. Nucl. Phys. 45 (1987) 522.

[49] G. Martinelli, plenary talk given at PANIC96, 22-28 May 1996, Williamsburg (USA), to appear in the Proceedings.

[50] M. Gronau, talk given at this workshop, hep-ph9609430 and refs. therein.

[51] J. Chay, H. Georgi and B. Grinstein, Phys. Lett. B247 (1990) 399.

[52] I.I. Bigi, N.G. Uraltsev and A.I. Vainshtein, Phys. Lett. B293 (1992) 430; E: B297 (1993) 477; I.I. Bigi, M.A. Shifman, N.G. Uraltsev and A.I. Vainshtein, Phys. Rev. Lett. 71 (1993) 496, Phys. Lett. B328 (1994) 431 and Int. J. Mod. Phys. A9 (1994) 2467; I.I. Bigi et al., Proceedings of the Annual Meeting of the Division of Particles and Fields of the APS, Batavia, Illinois, 1992, C. Albright et al. eds. (World Scientific Singapore, 1993), p. 610 .

[53] A.V. Manohar and M.B. Wise, Phys. Rev. D49 (1994) 1310.

[54] M. Neubert, Phys. Rev. D49 (1994) 3392 and 4623; T. Mannel and M. Neubert, Phys. Rev. D50 (1994) 2037.

[55] M. Neubert and C.T. Sachrajda, CERN-TH/96-19, hep-ph/9603202 and refs. therein.

[56] I.I. Bigi, M.A. Shifman, N.G. Uraltsev and A.I. Vainshtein, Phys. Rev. D50 (1994) 2234. 
[57] M. Beneke and V.M. Braun, Nucl. Phys. B438 (1994) 301.

[58] M. Beneke, V.M. Braun and V.I. Zakharov, Phys. Rev. Lett. 73 (1994) 3059.

[59] A.H.Mueller, Nucl. Phys. B250 (1985) 327; Phys. Lett. B308 (1993) 355; Proceedings of the Workshop "QCD: 20 years Later", Aachen, June 1992 eds. P.M.Zerwas and H.A.Kastrup (World Scientific, Singapore, 1993) p. 162.

[60] M. Neubert, Phys. Rev. D46 (1992) 1097.

[61] E. Bagan, P. Ball, V.M. Braun and H.G. Dosch, Phys. Lett. B278 (1992) 457.

[62] A.F. Falk, M. Luke and M.J. Savage, Phys. Rev. D53 (1996) 6316.

[63] M. Gremm, A. Kapustin, Z Ligeti and N.B. Wise, Phys. Rev. Lett. 77 (1996) 20.

[64] M. Crisafulli, V. Giménez, G. Martinelli and C.T. Sachrajda, Nucl. Phys. B457 (1995) 594; V. Giménez, G. Martinelli and C.T. Sachrajda, CERN-TH-96-163, heplat/9607018.

[65] V. Eletsky and E. Shuryak, Phys. Lett. B276 (1992) 191.

[66] P. Ball and V.M. Braun, Phys. Rev. D49 (1994) 2472.

[67] I. Bigi, M.A. Shifman, N.G. Uraltsev and A. Vainshtein, Phys. Rev. D52 (1995) 196.

[68] A. Kapustin, B. Grinstein, Z. Ligeti and N.B. Wise, Phys. Lett. B375 (1996) 327.

[69] Z. Ligeti and Y. Nir, Phys. Rev. D49 (1994) 4331.

[70] V. Giménez, G. Martinelli, C.T. Sachrajda, FTUV-96-49, hep-lat/9608039 and CERNTH-96-175, hep-lat/9607055.

[71] V. Chernyak, BUDKERINP-96-23, hep-ph/9604376.

[72] M. Neubert, CERN-TH/96-208, hep-ph/9608211.

[73] N. Cabibbo and L. Maiani, Phys. Lett. B79 (1978) 109.

[74] A. Ali and E. Pietarinen, Nucl. Phys. B154 (1979) 519.

[75] G. Altarelli, N. Cabibbo, G. Corbò, L. Maiani and G. Martinelli, Nucl. Phys. B208 (1982) 365. 
[76] B. Guberina, S. Nussinov, R. Peccei and R. Rücl, Phys. Lett. B89 (1979) 111; N. Bilic, B. Guberina and J. Trampetic, Nucl. Phys. B248 (1984) 261; B. Guberina, R. Rücl and J. Trampetic, Z. Phys. C33 (1986) 297; M.A. Shifman and M.B. Voloshin, Sov. J. Nucl. Phys. 41 (1985) 120 and JEPT 64 (1986) 698; G. Altarelli and L. Maiani, Phys. Lett. 118B (1982) 414.

[77] I.I. Bigi, B. Blok, M.A. Shifman and A.I. Vainshtein, Phys. Lett. B323 (1994) 408.

[78] E. Bagan, P. Ball, V.M. Braun and P. Gosdzinsky, Phys. Lett. B342 (1995); ERRATUMibid. B374 (1996) 363.

[79] G. Altarelli, G. Martinelli, S. Petrarca and F. Rapuano, Phys. Lett. B382 (1996) 409.

[80] P. Colangelo, F. De Fazio, BARI-TH-96-230, hep-ph/9604425.

[81] B. Chibisov, R.D. Dikeman, M.A. Shifman and N. Uraltsev, TPI-MINN-96-05-T, hepph/9605465.

[82] M. Ciuchini, E. Gabrielli and G.F. Giudice, CERN-TH-96-073, hep-ph/9604438.

[83] M. Neubert, Proceedings of the Beijing Lepton-Photon Conference 1995, CERN-TH/95307, hep-ph/9511409 and refs. therein.

[84] F. Bedeschi, Proceedings of the Rencontres de la Vallee d'Aoste, La La Thuile, 1996.

[85] Particle Data Group, Phys. Rev. D50 (1994) 1174.

[86] E687 collaboration, P.L. Frabetti et al, Phys. Lett. B357 (1995) 678; WA89 collaboration, M.I. Adamovich et al., Phys. Lett. B358 (1995) 151; S. Ratti, Proceedings of the Rencontres de la Vallee d'Aoste, La Thuile, 1996.

[87] I.I. Bigi, University of Notre Dame Preprint UND-HEP-95-BIG02, hep-ph/9508408 and refs. therein.

[88] E.C. Poggio, H.R. Quinn and S. Weinberg, Phys. Rev. D13 (1976) 1958.

[89] M.A. Shifman, Talk given at PASCOS/HOPKINS 1995, Baltimore, 22-25 Mar 1995, TPI-MINN-95/15-T, hep-ph/9505289. 